\title{
Free-Form Splines Combining NURBS and Basic Shapes
}

\author{
Kȩstutis Karčiauskas ${ }^{1}$ and Jörg Peters ${ }^{2}$ \\ ${ }^{1}$ Vilnius University ${ }^{2}$ University of Florida
}

September 27, 2011

\begin{abstract}
We show how to combine, into one unified spline complex, $C^{2}$ tensorproduct bi-cubic NURBS and $G^{2}$ bi-cubic rational splines. The $G^{2}$ splines are capable of exactly representing basic shapes such as (pieces of) quadrics and surfaces of revolution, including tori and cyclides. The main challenge is to bridge the differing continuity. We transform the $G^{2}$ splines to splines that are $C^{2}$ in homogeneous space. This yields Hermite data for a transitional strip of tensor-product splines of degree $(6,5)$ that guarantees overall curvature continuity. Key to the construction is the $C^{2}$ parameterization of circles in homogeneous space. We also cover the simpler $G^{1}$ to $C^{1}$ transition.
\end{abstract}

\section{Motivation}

Applications, such as ball and socket joints, require designs to locally exactly reproduce parts of basic shapes, such as spheres, or more generally quadrics and rotational objects. Inclusion of such basic shapes can also serve to define and, for their extent within the design surface, guarantee fair surface shape. At the same time, downstream processing of the resulting design surfaces favors representation of basic shapes and $C^{2}$ splines in one standard form, namely as NURBS of moderate degree. Many downstream problems can be avoided if the combined NURBS complex, of basic shapes and free-form tensor-product splines, is one consistent structure without the piecemeal stitching by separate blends and fillets. 
This challenge has motivated our recent work [KP11c, KP11b, KP11a] whose constructions are based on a fresh look of both the theory of rational geometric splines and reproducing conics, circles in particular. Rational geometric splines have been developed as early as [Boe87, GB88] - but this classical work focused on algebraic generality and fails to provide constructive recipes. In particular, it does not address the challenge of setting the many scalar degrees of freedom of geometric continuity and rational weights that determine the quality of the resulting curves and surfaces. On the other hand, the piecemeal reproduction of conic segments by rational pieces in Bernstein-Bézier form (see e.g. [Far88, PBP02]) lacks the built-in smoothness required of a unified structure with unique control points. The trilogy [KP11c, KP11b, KP11a] provides building blocks for reproducing multiple basic shapes and automatically joining them smoothly, both for curves and surfaces. It offers $G^{1}$ continuous constructions of degree (bi-)2, and $G^{2}$ continuity of degree (bi-)3; the scalars defining geometric continuity and rational weights are algorithmically initialized to reproduce the basic shapes.

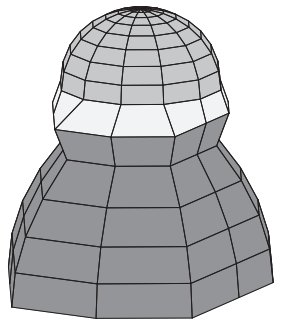

(a) combined mesh

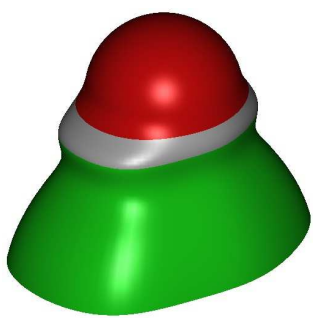

(b) combined surface

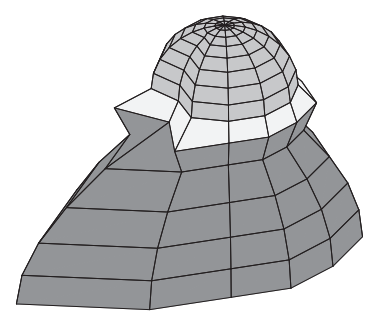

(c) edited mesh

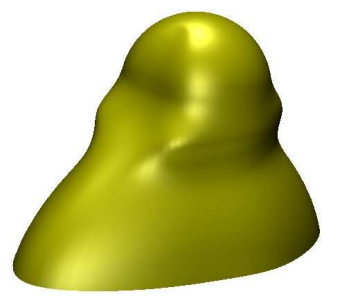

(d) $C^{2}$ ensemble

Figure 1: Sphere to NURBS transition. (a) The light gray control structure defines a spherical dome, the dark gray structure a $C^{2}$ spline. (b) The combined $C^{2}$ NURBS complex provides an automatic transition from the dome (red) with its spherical top and the spline (green) via a transition strip. (c) The resulting mesh can be freely modified as a rational spline resulting in the surface (d).

The main contribution of the present paper is to add to this framework an automatic and structurally efficient curvature-continuous transition (the gray strip in Fig. 1b) between standard $C^{2}$ bi-3 tensor-product free-form splines (green) and the basic shape (red). Note that the unified control net, behaves again like a NURBS control net (cf. Fig. 1c,d). The underlying challenge is to join freeform splines and basic surfaces when their geometric continuity differs in the direction parallel to the interface and hence a direct merging of bi-3 pieces as in [KP11b] is not possible. Fig. 2a illustrates that direct second-order Hermite 
interpolation of the boundaries of the two spline complexes does not preserve surface quality. This justifies our more elaborate approach. We extend ideas for $C^{2}$ circle parameterization in [KP11c] and [BP97] to convert $G^{2}$ to $C^{2}$ joins and then use the resulting Hermite data to automatically generate a transition strip as in Fig. 2b.

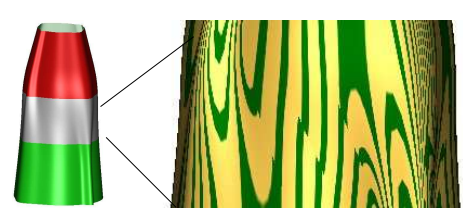

(a) naive Hermite interpolation

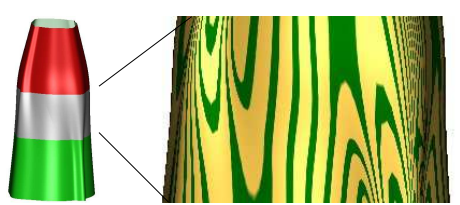

(b) technique of this paper

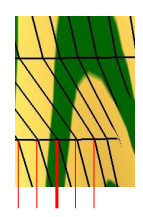

Figure 2: Failure of naive Hermite interpolation. (a) Discontinuous reflection lines when directly interpolating the existing spline complexes (red,green). The enlargement (right) shows additionally the control net (black) and the nonmatching $\beta_{i}$ (top vs. bottom vertical red line segment spacing; thicker at boundary control point). (b) Smooth reflection lines of our unified representation.

Outline In Section 2, we review the constructions that allow basic shapes to be part of a modifiable curvature-continuous rational bi-3 spline complex; and we explain why we use a parameterization of degree 6 that is guided by a degree 3 control structure. In Section 3, we introduce the reparameterization that allows us to transform, in Section $4, G^{2}$ splines of degree bi-3 as $C^{2}$ splines of degree 6 . Section 4 explains how to join these degree 6 splines with standard bi-3 NURBS to form a unified spline complex. Section 4 also provides several examples of unified $C^{2}$ spline complexes. In Section 5, we briefly sketch the much simpler unification of $G^{1}$ splines with bi-2 $C^{1}$ NURBS. Explicit formulas for the reparameterizations are listed in the Appendix.

\section{Rational representation of circles, of basic shapes and spline constructions}

Section 3.1 of [KP11b] gives a recipe for representing a wide variety of basic shapes in homogeneous form, as 4-tuples of trigonometric polynomials to represent in particular their prominent rotational features. These basic shapes include 
quadrics, with or without Möbius transformation, and generalized tori such as cyclides. A rational piecewise degree 3 model of the circle translates these definitions of basic shapes from a trigonometric to a rational polynomial $G^{2}$ continuous representation. Moreover, the representations are tensor-products so that we can represent each entry of the 4-tuple as a bi-3 tensor-product rational function in Bernstein-Bézier form (cf. [Far88, PBP02]):

$$
f(u, v):=\frac{\sum_{r=0}^{3} \sum_{s=0}^{3} w_{r s} b_{r s} B_{r}(u) B_{s}(v)}{\sum_{r=0}^{3} \sum_{s=0}^{3} w_{r s} B_{r}(u) B_{s}(v)} . \quad B_{k}(t):=\left(\begin{array}{l}
3 \\
k
\end{array}\right)(1-t)^{3-k} t^{k} .
$$

A key contribution of [KP11b] is to show how these tensor-product patches can equivalently be defined by a control structure consisting of: points in $\mathbb{R}^{3}$, weights and scalars of geometric continuity. This makes them part of a general curvaturecontinuous rational bi-3 spline complex, also derived in [KP11b]. Such a general bi-3 spline complex can be freely manipulated by modifying its control points, weights and scalars. The Bi-cubic Construction in [KP11b] provides explicit formulas for the spline surface in terms of the control structure. Together, this allows for a design work-flow that starts with the designer selecting basic shapes, an algorithm converting it to the control structure that the designer can manipulate and an automatic conversion of the modified control structure to a curvature continuous complex of surfaces.

Rational 4-tuples representing the basic shapes have a common denominator. While the common denominators cancel when projecting to $R^{3}$, their existence is crucial to establishing first $G^{2}$ continuity and then transforming to $C^{2}$ continuity of the rational 4-tuples in homogeneous space. We can place this denominator into a fifth slot so that all five coordinates are polynomial. In our constructions, the fifth slot entry is 1 if the shape was expressible as a rational spline to start with, as is the case for all conics except the closed circle. Otherwise, e.g. when constructing tori, cyclides and quadrics, the fifth slot depends on the denominator of the circle representation (2) and we call the representation homogeneous rational.

The circle representation therefore merits special attention. Circle arcs $f_{i}$ with opening angles $\alpha_{i}$ can be represented as rational cubic spline pieces with endweights equal to 1 and end-points $\mathbf{b}_{3}^{i-1}=\mathbf{b}_{0}^{i}$ on the circle:

$$
\begin{aligned}
f_{i} & :=\frac{\mathbf{b}_{0}^{i} B_{0}+w \mathbf{b}_{1}^{i} B_{1}+w \mathbf{b}_{2}^{i} B_{2}+\mathbf{b}_{3}^{i} B_{3}}{B_{0}+w B_{1}+w B_{2}+B_{3}}, \\
w & :=\frac{1}{3}+\frac{2}{3} \cos \frac{\alpha_{i}}{2} .
\end{aligned}
$$


The pieces meet up with second order, or $G^{2}$ Geometric Continuity. By definition $f_{i-1}:[0 . .1] \rightarrow \mathbb{R}^{d}$ and $f_{i}:[0 . .1] \rightarrow \mathbb{R}^{d}$, join $G^{1}$ at a common point $f_{i-1}(1)=f_{i}(0)$ if for some scalar $\beta_{i}>0$

$$
f_{i}^{\prime}(0)=\beta_{i} f_{i-1}^{\prime}(1)
$$

and $G^{2}$ if additionally there exist $\gamma_{i} \in \mathbb{R}$

$$
f_{i}^{\prime \prime}(0)=\beta_{i}^{2} f_{i-1}^{\prime \prime}(1)+\gamma_{i} f_{i-1}^{\prime}(1)
$$

The curve pieces defined by (2) join with $G^{2}$ continuity in each of the two coordinates with scalars

$$
\beta_{i}=\frac{\sin \frac{\alpha_{i}}{2}}{\sin \frac{\alpha_{i-1}}{2}}, \gamma_{i}=2 \beta_{i}\left(\tan \frac{\alpha_{i-1}}{4}+\tan \frac{\alpha_{i}}{4}\right) \sin \frac{\alpha_{i}}{2} .
$$

When $\beta_{i}=1$ and $\gamma_{i}=0$ in (3) and (4), the pieces are said to join (parametrically) $C^{2}$.

The homogeneous $C^{2}$ representation of a circle must be of degree at least 6 according to [BP97] implying parameterizations of degree at least 6 for the pieces of the basic shapes in the free-form spline complex. We now explain why we do not use such a parameterization of degree 6 directly, but have it guided by a degree 3 control structure. A degree $6 C^{2}$ spline can be expressed in B-spline form with 4 -fold knots. If adjacent spline pieces have knot intervals with ratio $\beta$ then we can encode this ratio by choosing $\beta$ as the parameter of geometric continuity [Joe89, Bar93]. If we represent adjacent pieces of the $C^{2}$ spline in Bézier form, their Bézier coefficients $\mathbf{b}_{k}$ and $\overline{\mathbf{b}}_{k}$ are related by

$$
\begin{gathered}
\mathbf{P}:=(\beta+1) \mathbf{b}_{5}-\beta \mathbf{b}_{4}=\frac{\beta+1}{\beta} \overline{\mathbf{b}}_{1}-\frac{1}{\beta} \overline{\mathbf{b}}_{2}=: \overline{\mathbf{P}} \\
\mathbf{b}_{5}:=\frac{\beta \mathbf{b}_{4}+\mathbf{P}}{\beta+1}, \overline{\mathbf{b}}_{1}:=\frac{\beta \mathbf{P}+\overline{\mathbf{b}}_{2}}{\beta+1} .
\end{gathered}
$$

The Bézier coefficients $\mathbf{b}_{2}, \mathbf{b}_{3}, \mathbf{b}_{4}$ and $\overline{\mathbf{b}}_{2}, \overline{\mathbf{b}}_{3}, \overline{\mathbf{b}}_{4}$ are unconstrained and coincide with the control points of the degree $6 \mathrm{~B}$-spline representation; $\mathbf{P}$, also free to choose, is an additional B-spline control point. However, as Fig. 4 illustrates, these B-spline control points modify the curve much below the scale of individual spline pieces. We think this makes them unsuited as direct design handles; it is cumbersome to replicate a shape like Fig. 4a. Rather than as a constructive device, we will therefore use the $C^{2}$ sextic spline as a dependent representation: Our 


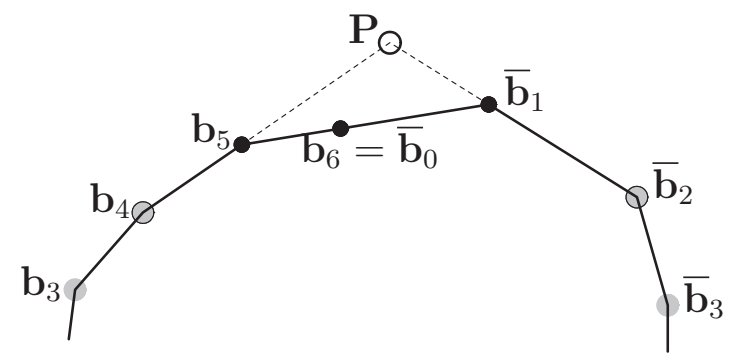

Figure 3: $C^{2}$ join of degree 6 curves according to (5), (6).

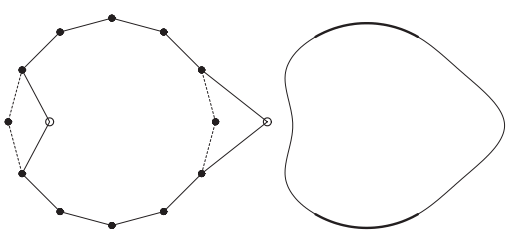

(a) $G^{2}$ degree 3

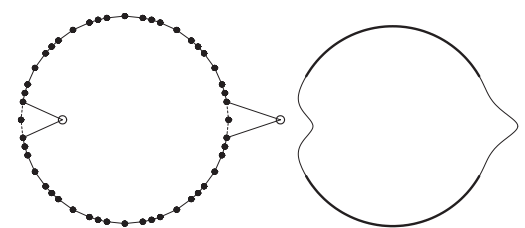

(b) $C^{2}$ degree 6

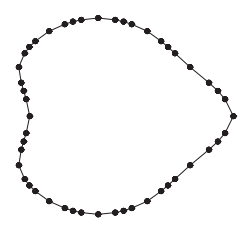

(c) derived $C^{2}$ spline

Figure 4: Free-form design starting from a circle. (a) Moving two control points (black disks to hollow disk) of a uniformly partitioned cubic $G^{2}$ spline yields a design whose thick segments remain exactly on the circle. (b) Control points of a uniformly partitioned sextic $C^{2}$ spline yield control at a much finer level than the spline pieces. (c) Control points of a sextic $C^{2}$ spline derived by the procedure in Section 4.1 from the $G^{2}$ cubic spline in (a).

construction in Section 4.1 transforms the cubic $G^{2}$ spline to a sextic $C^{2}$ spline, offering as design handles the better-spaced control points of the original cubic. This yields e.g. the sextic shown in Fig. 4c. This explains why, in the following, we do not simply replace the trigonometric expressions of the basic shapes by $C^{2}$ sextics and then Hermite-interpolate the resulting $C^{2}$ splines. While simple, this approach does not afford the familiar bi-3 NURBS or bi-3 $G^{2}$ rational spline manipulation illustrated in Fig. 1c, 4c, 9c, 10c.

\section{Rational cubic reparameterizations of the circle}

The goal is to merge standard $C^{2}$ bi-3 tensor-product free-form splines and fragments of basic shapes expressed as rational bi-3 $G^{2}$ splines. Our construction is based on re-parameterizing the $G^{2}$ circle parameterizations that are embedded into 
homogeneous rational polynomials when translating sin and cos terms in the basic shapes' definition into a homogeneous rational form - cf. Section 2. The $G^{2}$ representation will be replaced by a $C^{2}$ representation in homogeneous space. We will use two different circle parameterizations with $C^{2}$ splines of degree 6 . The first is an extension of [KP11c], the second builds on the approach of [BP97]. For a uniform partition of the circle, both yield the same cubic and hence the same sextic $C^{2}$ presentation of a circle. (In fact, for uniform partitions there is yet another option: composition with the quadratic map of [KP11c, Section 8] followed by multiplication of the homogeneous quartic by $(1-u)^{2}+2(3-(1+\cos (\alpha / 2)) \nu)(1-u) u+u^{2}$ for a $\nu$ defined in [KP11c, Section 8]. This does not contradict the claim of uniqueness in [BP97] which relies on the assumption that the coordinate functions have no common divisor. )

Ternary-split parameterization $\rho$. For the first circle parameterization, we consider consecutive circular $\operatorname{arcs} f^{i}$ with opening angle $\alpha_{i}$ and newly choosable geometric continuity scalars $\tilde{\beta}_{i}$ between arcs $f^{i-1}$ and $f^{i}$. (Heuristically, it is good to preserve the original parameterization of the basic shapes as much as possible, i.e. set $\tilde{\beta}_{i}:=\beta_{i}$.) We reparameterize $f^{i}$ by a three-split rational cubic with segments $\rho^{i, j}:[0,1] \rightarrow \mathbb{R}, j=0,1,2$. The coefficients of this $C^{2}$-connected triplet of arcs are defined in Section 7.1. (For a uniform partition and all $\tilde{\beta}_{i}=1$, a single piece suffices.) By construction, the pieces $f^{i} \circ \rho^{i, j}, j=0,1,2$ of one $\operatorname{arc}$ join $C^{2}$. Analogous to [KP11c], the following lemma asserts $C^{2}$ continuity also between adjacent rational arcs, not just internally.

Lemma 1 (homogeneous $C^{2}$ reparameterization for circles) Let $f^{i} \circ \rho^{i, j}, j=$ $0,1,2$ be the three-piece reparameterization of the circular arc $f^{i}$. Then the sextics $f^{i-1}\left(\rho^{i-1,2}\right)$ and $f^{i}\left(\rho^{i, 0}\right)$ are $C^{2}$-connected in homogeneous space with scalar $\tilde{\beta}_{i}$.

Numerical Parametrization $\sigma$. For our second reparameterization consider the partition by points $\mathbf{p}_{i}$ (black disks) of the unit circle with center $O=(0,0)$ in Fig. 5a. These $\mathbf{p}_{i}$ project on the Northern semi-circle with center $C=(0,-1)$ by intersecting the semi-circle with the line through $C$ and $\mathbf{p}_{i}$ :

$$
\mathcal{P}(x, y):=(c x, c-1+c y), \quad c:=\frac{1}{\sqrt{x^{2}+(y+1)^{2}}} .
$$

(As noticed in [BP97], if the unit circle partition is rotated by $\alpha$ then the projected partition is rotated by $\alpha / 2$.) The intersections with the blue 'Southern' semicircle are useful to complete a circle. We interpolate the projections $\overline{\mathbf{p}}_{i}$ by a cubic 


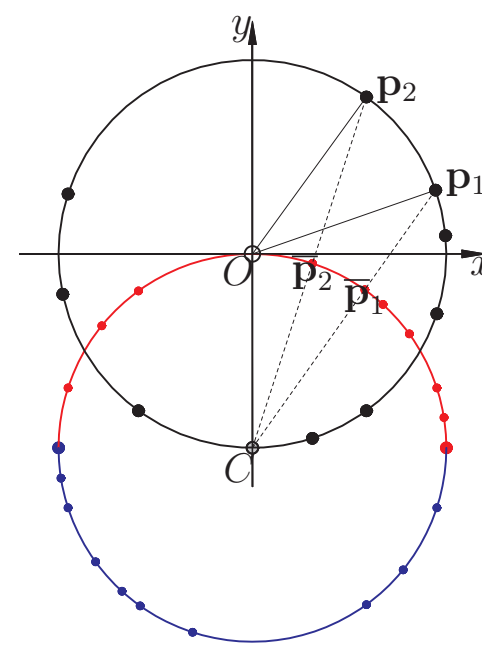

(a) Projection $\mathcal{P}$

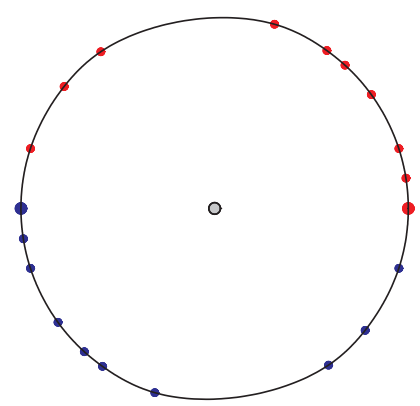

(b) $C^{2}$ spline interpolant

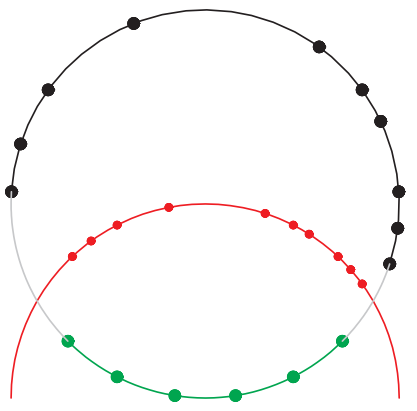

(c) partial interpolation

Figure 5: $C^{2}$ circle parameterization. (a) The projection of [BP97]. (b) A cubic $C^{2}$ spline interpolating the projected points ( this is not a circle!). (c) Projection $\mathcal{P}$ applied only to non-uniform parts (black) of the partition, not to the uniform (green) part.

$C^{2}$ spline with scalars $\tilde{\beta}_{i}$ (cf. Fig. 5b), solving a tridiagonal linear systems of equations. Then we project the result back to the black circle by

$$
\mathcal{P}^{-1}:(x, y) \rightarrow\left(2 x(1+y),(1+y)^{2}-x^{2}, x^{2}+(y+1)^{2}\right) .
$$

This yields a $C^{2}$ sextic homogeneous parameterization of the circle. Finally, for each segment and its given quadratic $q$, we calculate $\sigma$ so that $q \circ \sigma$ equals the sextic. The original knot spacings, i.e. $\tilde{\beta}_{i}=\beta_{i}$, work well. Where we use other choices, we do not deviate by much since this can lead to non-monotone parameterizations.

The best of the two approaches. The ternary-split $\rho$ triples the number of segments - but this is not visible to the designer. In favor of $\rho$, its formulas are explicit, while $\sigma$ requires solving a system of equations that depend on the partition. This is a major drawback of $\sigma$ since uniform or symmetric subsequences in an overall non-uniform circle partition are not preserved. Since $\rho$ preserves uniformity and symmetry, we combine the approaches by applying $\rho$ to the uniform or symmetric parts and compute $\sigma$ for the remainder from smaller tridiagonal sys- 
tems, e.g. for the red sequence in Fig. 5c. For the transition between $\rho$ and $\sigma$ (from the upper black to the lower green in Fig. 5c), Section 7.2 lists explicit formulas.

Given the $C^{2}$-parameterized circles, we use the formulas of [KP11c, Lemma 1] and Section 7.2 to verify by symbolic computation that the reparameterization transforms $G^{2}$ transitions to $C^{2}$ transitions in homogeneous space.

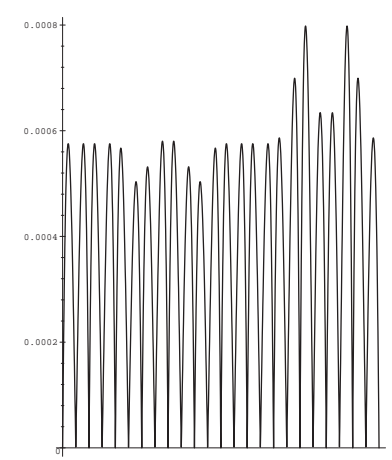

(a) parametric difference

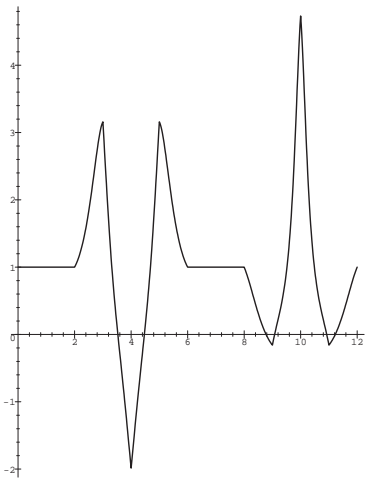

(b) curvature of Fig. 4a

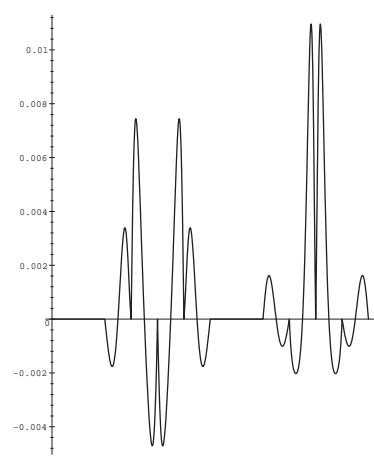

(c) curvature difference

Figure 6: Perturbation of non-basic shape when changing to degree 6. (a) Parametric difference between cubic $G^{2}$ spline of Fig. 4a (perturbed unit circle) and its derived $C^{2}$ sextic shown in Fig. 4c. (b) Curvature plot of Fig. 4a. (c) The difference in curvature between the cubic and the sextic spline is in the 1 percent range.

\section{Combining NURBS and $G^{2}$ splines}

Reparameterizing from $G^{2}$ to $C^{2}$ is a subtle task when we want to preserve the basic shapes and overall fairness. We will transform the $G^{2}$ spline so that fragments of basic shapes are exactly preserved. Elsewhere, to keep the overall polynomial degree low, the new surface will differ ever-so-slightly from the original bi-cubic surface, e.g. the non-spherical base of the (red) dome in Fig. 1b. For a 2D curve, this difference is quantified in Fig. 6: it is difficult to spot even the change in curvature. 


\subsection{Switching from $G^{2}$ to $C^{2}$ spline curves}

Since we construct surfaces as tensor-products, we first focus on the univariate case, i.e. curves. Recall that the circle parameterization translates trigonometric to a rational polynomial representation of the basic shapes. In [KP11b] the circle parameterization was chosen to consist of $G^{2}$-connected pieces of degree 3 (cf. Section 2). The main task is to replace these rational $G^{2}$ cubic circle parameterizations in the definition of the basic shapes to homogeneous $C^{2}$ parameterizations. To this end, we reparameterize the circle using $r:=\frac{\bar{r}}{r}$ where $r$ is one of $\rho, \sigma$ or their transition constructed in Section 3. The composition of the cubic spline with $r$ is of degree 9. We reduce the degree by instead composing $r$ with two Bézier polynomials of degree 2 that match the cubic's expansion up to order 2 at either end point. Composing $r$ of degree 3 with a Bézier polynomial of degree 2 (with coefficients $\mathbf{p}_{k}$ and weights $w_{k}$ ) yields, after dropping common denominators, the following polynomial expansion of degree 6 :

$$
q:=\left(\sum_{k=0}^{2} w_{k} \mathbf{p}_{k}\left(\begin{array}{l}
2 \\
k
\end{array}\right)(\underline{r}-\bar{r})^{2-k} \bar{r}^{k}, \sum_{k=0}^{2} w_{k}\left(\begin{array}{l}
2 \\
k
\end{array}\right)(\underline{r}-\bar{r})^{2-k} \bar{r}^{k}\right) .
$$

Here is a good point to recall that the constructions do not mix coordinates and hence we treat each coordinate one at a time. That is (9) represents one of the four coordinates. The rational expression of the coordinate is in turn written as a 2-tuple in homogeneous 1-space: $\mathbf{p}_{k} \in \mathbb{R}$. The last coordinate in (9), i.e. the second sum, is common to all four homogeneous coordinates. This common slot, corresponding to the denominator of rational expressions and representable as a fifth polynomial slot, will be eliminated when the 4 homogeneous coordinates are projected to 3 -space.

Let $r^{i}$ be assigned to the $i$-th segment of a cubic $G^{2}$ spline. Where the spline is already $C^{2}$, and by default for transition segments, $r^{i}$ is the identity map.

For $r^{i}=\sigma$, let $f^{i, 0}$ be the quadratic in Bézier form that matches the $G^{2}$ cubic circle piece $f^{i}$ up to the second derivative at 0 (blue curve segment in Fig. 7 (left)) and $\dot{f}^{i, 1}$ the quadratic matching $f^{i}$ at 1 (green). The composition $g^{i, 0}:=\dot{f}^{i, 0} \circ r^{i}$ yields sextic control points $\mathbf{b}_{k}^{i, 0}$ and $g^{i, 1}:=\dot{f}^{i, 1} \circ r^{i}$ yields control points $\mathbf{b}_{k}^{i, 1}$, $k=0, \ldots, 6$. These are merged into one sextic $\mathbf{b}^{i}$ with control points

$$
\mathbf{b}_{k}^{i}:=\mathbf{b}_{k}^{i, 0}, k=0,1,2 ; \quad \mathbf{b}_{k}^{i}:=\mathbf{b}_{k}^{i, 1}, k=4,5,6 ; \quad \mathbf{b}_{3}^{i}:=\frac{\mathbf{b}_{3}^{i, 0}+\mathbf{b}_{3}^{i, 1}}{2} .
$$

Where $r^{i}$ is the identity, the composition amounts to degree-raising and the spline remains $C^{2}$. Where the segment is a $G^{2}$ cubic homogeneous rational spline piece, 

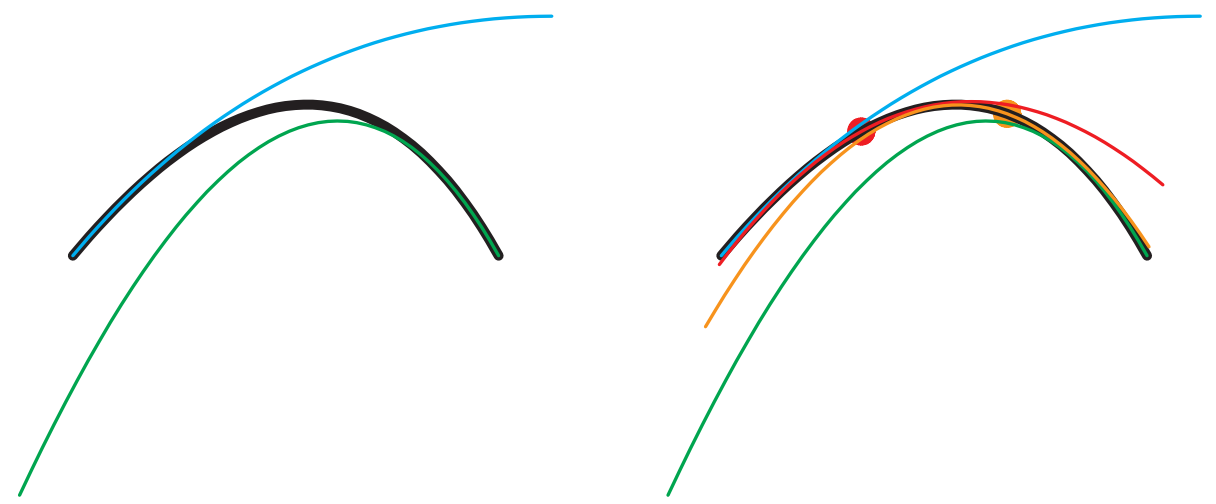

Figure 7: Connecting quadratic Hermite interpolants. (left) for $\sigma$; (right) for $\rho$, consisting of three pieces.

$r^{i}$ has been constructed to yield $C^{2}$-connected pieces of degree 6 and basic shapes are preserved. Only at the interface between such a sextic spline with control points $\mathbf{b}_{j}$ and a degree-raised piece with control points $\overline{\mathbf{b}}_{j}$ is the transition just $C^{0}$ in homogeneous space. Since, at the transition, we do not reproduce basic shapes anyhow, we adjust the points as follows to obtain a $C^{2}$ transition there too. With $\mathbf{P}$ and $\overline{\mathbf{P}}$ from (5),

$$
\mathbf{P}^{\text {new }}:=\frac{\mathbf{P}+\overline{\mathbf{P}}}{2}, \quad \mathbf{b}_{5}, \overline{\mathbf{b}}_{1} \text { defined by (6), } \quad \mathbf{b}_{6}=\overline{\mathbf{b}}_{0}:=\frac{\beta \mathbf{b}_{5}+\overline{\mathbf{b}}_{1}}{\beta+1} .
$$

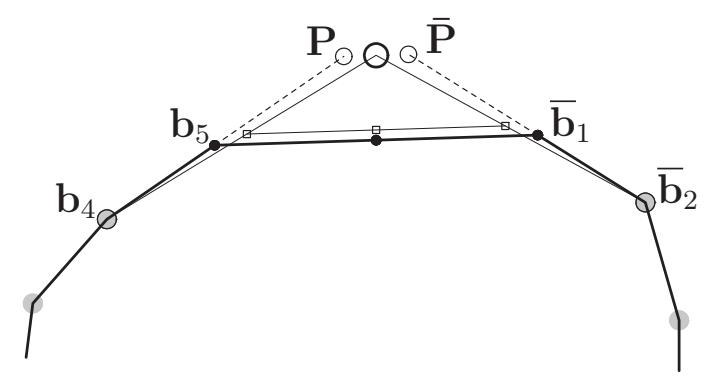

Figure 8: Adjustment of the $C^{2}$ join: small black disks are the original sextic control points, small boxes their adjustment.

Where $r=\rho$, we use two additional quadratics matching the original data at $u=\frac{1}{3}$ (red in Fig. 8b) and $u=\frac{2}{3}$ (gold) and derive from every consecutive pair a curve segment, as we did for the single segment and $\sigma$. 


\subsection{Switching from $G^{2}$ to $C^{2}$ spline surfaces}

With the re-parameterizations, we can now state the simple

\section{Algorithm for combining NURBS and $G^{2}$ splines.}

- Including the transition layer of the mesh (white in Fig. 1a), we replace $G^{2}$ splines by sextic $C^{2}$-joined splines by the technique in Section 4.1. Here, we may replace $G^{2}$ cubics by $C^{2}$ sextics in only one direction, i.e. along the interface, or in both.

- Both the NURBS surface (green in Fig. 1b) and the $C^{2}$ sextic spline (red) are now well-defined up to the transition and we can collect second order Hermite data (three layers of Bézier coefficients) across the transition boundary.

- Both the bi-3 NURBS Hermite data and the sextic spline data are degreeraised to $(6,5)$ to populate the two halves of patches of degree $(6,5)$ that form the transition (gray in Fig. 1b).

Examples. Fig. 1 shows a sphere and a tensor-product spline in unified representation. The continuity scalars $\beta$ of the sphere in circular direction agree with those of the tensor-product spline (both are 1). So it is tempting to directly Hermite-interpolate by a surface ring of piecewise degree $(3,5)$. Indeed, the loss of smoothness due to the mismatch in the rational component is hardly visible; but for non-matching $\beta$ the mismatch can be very apparent as in Fig. 2 a.

Fig. 9 shows a unified $C^{2}$ surface representing parts of a sphere and a $G^{2}$ spline. The $G^{2}$ spline itself is the result of merging a circular and two hyperbolic cylinders. The local adjustment (11) is already applied to the cylinder-ensemble (dark gray part of the mesh), in order to $C^{2}$-join the circular cylinder to the $C^{2}$ connected hyperbolic cylinders.

Fig. 10 shows how remapping to the sextic $C^{2}$ representation allows for additional flexibility for design starting with basic shapes. The hemi-sphere is joined with a $G^{2}$ spline derived from four circular cylinders. The scalars $\beta$ are all 1 , but the scalars $\gamma$ and the rational weights do not agree.

Fig. 11 shows a unified representation of two differently partitioned and perturbed cones. Creases of different sharpness meet up. At the transition we choose $\tilde{\beta}_{i}:=\sqrt{\beta_{i}^{\text {top }} \beta_{i}^{\text {bottom }}}$ since $\beta_{i}^{\text {top }}$ and $\beta_{i}^{\text {bottom }}$ differ strongly (see Fig. 2a). 


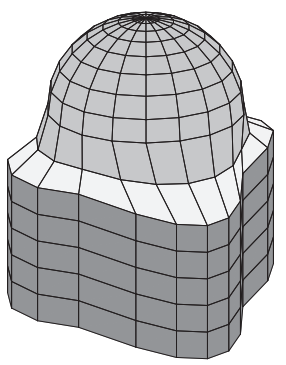

(a) combined mesh

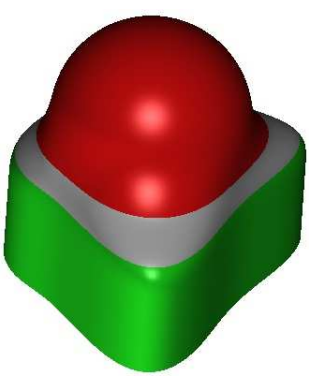

(b) combined surface

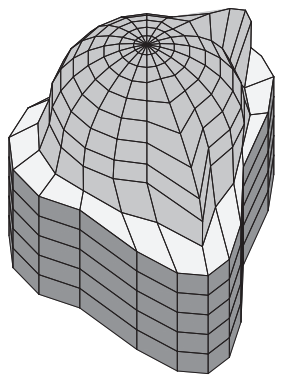

(c) edited mesh

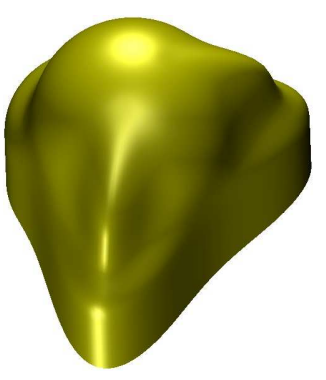

(d) $C^{2}$ ensemble

Figure 9: Sphere and cylinders with free-form spline transition. (a) The light gray control structure defines a dome with spherical top. The dark gray structure itself combines a circular with two hyperbolic cylinders. (b) $C^{2}$ NURBS complex with gray transition from the dome (red) to the cylinders (green). (c) The resulting mesh can be freely modified as free-form spline yielding the sextic $C^{2}$ surface (d).

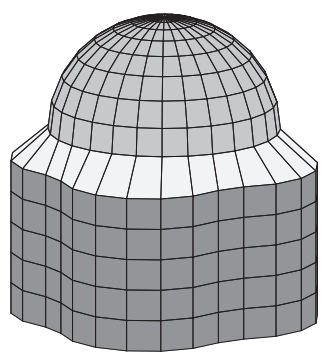

(a) combined mesh

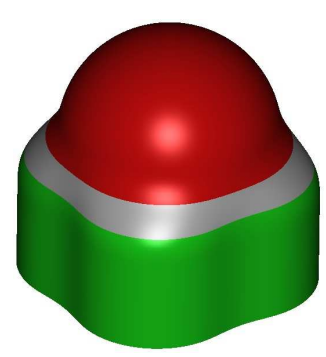

(b) combined surface

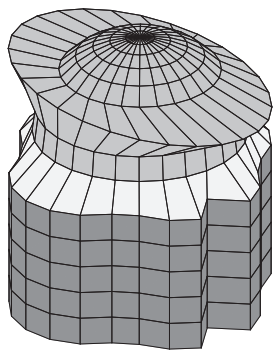

(c) design variant

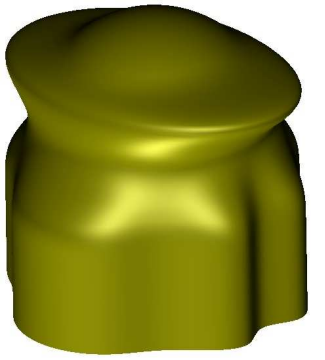

(d) $C^{2}$ ensemble

Figure 10: Increased flexibility after transformation to the unified $C^{2}$ degree 6 representation. (a) Spherical dome (light gray then top of red) and four circular cylinders (dark gray then green) define the $C^{2}$ surface (b). Replacing both surface pieces by the $C^{2}$ degree 6 representation allows for (c) free-form design resulting in the NURBS surface (d). 


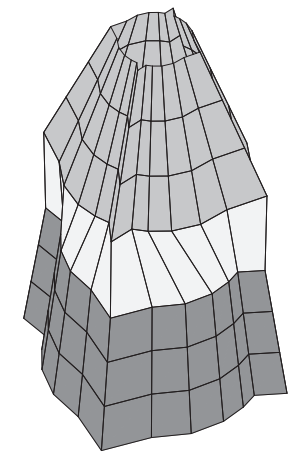

(a) unified control net
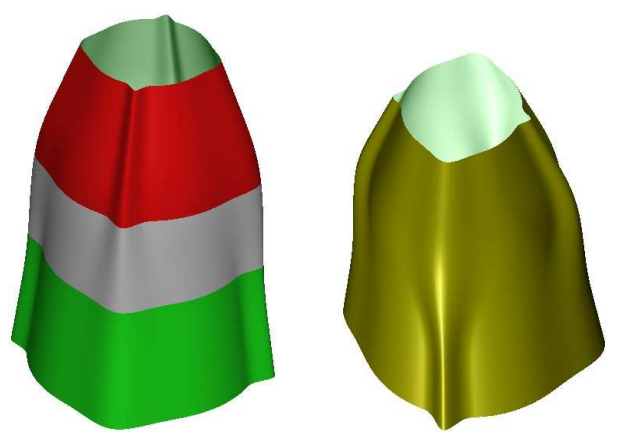

(b) unified surface - two views

Figure 11: Spline surface unifying two $G^{2}$ rational splines (light, dark gray) defining two differently creased cones.

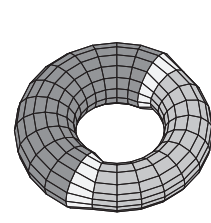

(a) mesh

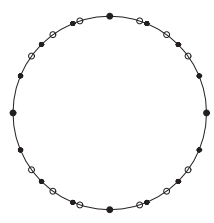

(b) 2 partitions

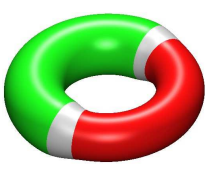

(c) unified spline

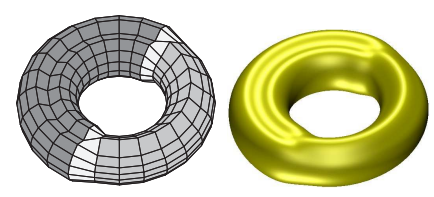

(d) design variant

Figure 12: Merging tori with differing cross-sectional partitions (gray vs black disks in (b)).

Fig. 12 shows a unified representation of two $G^{2}$ tori. Our approach of converting both to $C^{2}$ bi-sextics avoids knot insertion when matching pieces along the transition. As in Fig. 11, we average $\beta_{i}^{\text {top }}$ and $\beta_{i}^{\text {bottom }}$.

\section{Combining NURBS and $G^{1}$ splines}

To combine NURBS of degree 2 with the $G^{1}$ basic-shape-reproducing splines of [KP11a] is a comparatively simpler task. Representation of the uniformly partitioned circle in homogeneous cubic $C^{1}$ form is already possible by replacing each quadratic segment of the $G^{1}$ spline by two $C^{1}$-connected cubics [KP11a, Section 7] or a single quartic segment [BP97].

For the general case, we define, analogous to the construction of $\rho$, for each segment, a rational quadratic reparameterization consisting of $C^{1}$-connected pieces $\eta^{0}, \eta^{1}$ defined in Section 7.3. Each quadratic segment $q^{i}$ is then replaced by two 
$C^{1}$-connected quartics $q^{i} \circ \eta^{i, 0}$ and $q^{i} \circ \eta^{i, 1}$. Then

$$
\ldots, q^{i-1} \circ \eta^{i-1,1}, q^{i} \circ \eta^{i, 0}, q^{i} \circ \eta^{i, 1}, \ldots
$$

form a piecewise degree $4 C^{1}$ spline that we can use to re-express (the acrossboundary 1-jet expansion of) the layer adjacent to the strip as $(4,2)$ splines that are homogenous $C^{1}$ and of degree 4 in the direction parallel to the strip. Each complex provides first-order Hermite data across the boundary. The double-patchwide transition strip of degree $(4,2)$ interpolates the Hermite data. Its centerline is determined by the constraint that the double strip be $C^{1}$.

Unlike the $G^{2}$ to $C^{2}$ transformation, this re-parameterization exactly reproduces the quadratic rational $G^{1}$ spline. Therefore no further adjustment is necessary. This localizes work to the transition strip.

Examples. Fig. 14 illustrates partial preservation and combination of torus pieces with differing $\beta_{i}$ spacing in one direction and T-junctions in the other direction. Fig. 15 illustrates unified structures of surfaces of revolution and free-form $C^{1}$ splines. By splitting the transversal 1-jet(s) to the finest partition on either side, the local transition accomodates different partitions in the two input structures (cf. the T-junctions in Fig. 15e).

\section{Summary}

We obtained a unified spline representation of bi-3 NURBS and $G^{2}$ bi-3 splines that reproduce basic shapes. This comes at the cost of replacing the degree $3 G^{2}$ splines by degree $6 C^{2}$ splines with more pieces. The basic shapes continue to be exactly represented; and we gain additional freedom in a unified control net that behaves like that of a regular bi-cubic spline. In the simpler $G^{1}$ to $C^{1}$ unification all work is local to the transition strip. While we can also localize the $G^{2}$ to $C^{2}$ transition, construction is more involved than the one presented.

\section{Acknowledgments.}

Work supported in part by NSF Grant CCF-1117695. 


\section{References}

[Bar93] BARSKY B. A.: Rational beta-splines for representing curves and surfaces. IEEE Comp Graph and Appl 13, 6 (1993), 24-32.

[Boe87] Boenm W.: Rational geometric splines. Computer Aided Geometric Design 4, 1-2 (July 1987), 67-77.

[BP97] Bangert C., Prautzsch H.: Circle and sphere as rational splines. Neural, Parallel and Scientific Computations, 5 (1997), 153-162.

[Far88] FARIN G.: Curves and Surfaces for Computer Aided Geometric Design: A Practical Guide. Academic Press, 1988.

[GB88] Goldman R. N., BARsky B. A.: Beta Continuity and Its Application to Rational Beta-splines. Technical Report CSD-88-442, University of California, Berkeley, Aug. 1988.

[Joe89] JoE B.: Multiple-knot and rational cubic beta-splines. ACM Transactions on Graphics 8, 2 (1989), 100-120.

[KP11a] KarČIAuskas K., Peters J.: Modeling with rational biquadratic splines. In SIAM GD/SPM conference (2011), Bajaj C., Kim M.-S., Hahmann S., (Eds.), pp. 1-10.

[KP11b] KarČIAUSKas K., Peters J.: Rational bi-cubic $G^{2}$ splines for design with basic shapes. Computer graphics forum 30, 5 (2011), 1389-1395.

[KP11c] KarČIAUskas K., Peters J.: Rational $G^{2}$ splines. Graphical Models 23, 5 (2011), 286-295.

[PBP02] Prautzsch H., Boehm W., Paluszny M.: Bézier and B-spline techniques. Springer Verlag, 2002.

\section{Appendix: Reparameterizations}

\subsection{Cubic $C^{2}$ ternary-split parameterization $\rho$}

Formulas (13) and (14) below enable the $C^{2}$ construction internal to the ternarysplit arcs. Given the newly choosable scalars of geometric continuity $\tilde{\beta}$ and the 
opening angles $\alpha_{i}$, we define $\tau_{i}:=\tan \frac{\alpha_{i}}{4}$, and, without loss of generality, focus on the segment $i=0$, omitting the index $i$ below. The homogeneous control points $\rho_{k}^{j}, k=0 \ldots 3, j=0,1,2$ of $\rho^{j}$ are defined as follows (cf. Fig. 13).

- We set

$$
\begin{aligned}
a_{-1}:= & \tau_{1}\left(1+\tau_{0}^{2}\right)+2 \tau_{0} \tau_{1}\left(\tau_{0}+\tau_{1}\right) \tilde{\beta}_{1}+\tau_{0}\left(1+\tau_{1}^{2}\right) \tilde{\beta}_{1}^{2}, \\
a_{0}:= & 2 \tau_{1}\left(\tau_{0}+\tau_{1}\right)+\left(3+4 \tau_{0} \tau_{1}+7 \tau_{1}^{2}\right) \tilde{\beta}_{1}+3\left(1+\tau_{1}^{2}\right) \tilde{\beta}_{1}^{2}, \\
a_{1}:= & 3\left(1+\tau_{0}^{2}\right)\left(1+\tau_{1}^{2}\right)+\left(6+10 \tau_{0}^{2}+8 \tau_{0} \tau_{1}+10 \tau_{1}^{2}+6 \tau_{0}^{2} \tau_{1}^{2}\right) \tilde{\beta}_{1} \\
& +3\left(1+\tau_{0}^{2}\right)\left(1+\tau_{1}^{2}\right) \tilde{\beta}_{1}^{2},
\end{aligned}
$$

and define

$$
d:=\frac{a_{-1}}{\tau_{0} a_{0}}, \nu:=\frac{\left(1+\tau_{0}^{2}\right)\left(1+\tilde{\beta}_{1}\right) a_{0}}{\tilde{\beta}_{1} a_{1}} .
$$

- We define a rational cubic map $h^{1}(u):[0,1] \rightarrow \mathbb{R}$ by homogeneous control points

$$
h_{0}^{1}:=\left[\begin{array}{l}
0 \\
1
\end{array}\right], h_{1}^{1}:=\left[\begin{array}{c}
\nu d \\
\nu
\end{array}\right], h_{2}^{1}:=\left[\begin{array}{c}
\nu(1-d) \\
\nu
\end{array}\right], h_{3}^{1}:=\left[\begin{array}{l}
1 \\
1
\end{array}\right],
$$

and a second, symmetric one, $h^{0}(u)$, by replacing in $h^{1}: \tau_{1} \rightarrow \tau_{-1}, \tilde{\beta}_{1} \rightarrow$ $1 / \tilde{\beta}_{0}$.

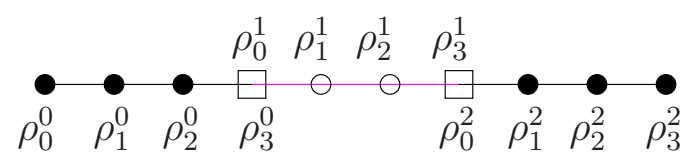

Figure 13: Coefficients of the rational cubic reparameterization $\rho$.

- We obtain the left piece of our reparameterization as $\rho^{0}(u):=h^{0}(u / 3)$ and the right as $\rho^{2}(u):=h^{1}(2 / 3+u / 3)$ with corresponding homogeneous control points $\rho_{k}^{0}, \rho_{k}^{2}$.

- We overwrite the end points $\rho_{3}^{0}$ and $\rho_{0}^{2}$ and set the remaining coefficients of the middle piece so that the three pieces join $C^{2}$ :

$$
\begin{gathered}
\rho_{3}^{0}:=-\frac{1}{3} \rho_{1}^{0}+\frac{7}{6} \rho_{2}^{0}+\frac{1}{3} \rho_{1}^{2}-\frac{1}{6} \rho_{2}^{2}, \quad \rho_{0}^{2}:=-\frac{1}{6} \rho_{1}^{0}+\frac{1}{3} \rho_{2}^{0}+\frac{7}{6} \rho_{1}^{2}-\frac{1}{3} \rho_{2}^{2}, \\
\rho_{0}^{1}:=\rho_{3}^{0}, \quad \rho_{1}^{1}:=2 \rho_{3}^{0}-\rho_{2}^{0}, \quad \rho_{2}^{1}:=2 \rho_{0}^{2}-\rho_{1}^{2}, \quad \rho_{3}^{1}:=\rho_{0}^{2} .
\end{gathered}
$$

For a uniform partition and all $\tilde{\beta}_{i}=1$, there is no need for such splitting since then $d$ and $\nu$ do not dependent on $i$. 


\subsection{Cubic $C^{2}$ transition reparameterization}

For two neighboring circular arcs with the opening angles $\alpha, \bar{\alpha}$. Let $\tau:=\tan (\alpha / 4)$, $\bar{\tau}:=\tan (\bar{\alpha} / 4)$. The homogeneous control points $h_{k}, \bar{h}_{k}, k=0, \ldots, 3$ of the reparameterizations $\rho, \bar{\rho}$ of the quadratic circular arcs yield a homogeneous $C^{2}$ join with parameter $\tilde{\beta}$ as follows, derived and verified by symbolic computation. We set

$$
\begin{aligned}
e_{1} & :=1+\tau^{2}, e_{2}:=1+\bar{\tau}^{2}, e_{3}:=-1+2 \tau \bar{\tau}+\tau^{2}, e_{4}:=-2 \tau(\tau+\bar{\tau}) \\
a_{1} & :=2\left(e_{1}+e_{3} \nu_{2}+e_{4} d_{2} \nu_{2}\right), a_{2}:=-e_{1}-e_{3} \nu_{1}-e_{4} d_{1} \nu_{1} \\
\bar{\nu}_{1} & :=\frac{2 e_{1}+a_{1} \tilde{\beta}}{2 e_{1}}, \bar{\nu}_{2}:=\frac{e_{1}+a_{1} \tilde{\beta}+\left(a_{1}+a_{2}\right) \tilde{\beta}^{2}}{e_{1}}, \bar{d}_{1}:=\frac{\tilde{\beta} \tau \nu_{2}\left(1-d_{2}\right) e_{2}}{e_{1} \bar{\tau} \bar{\nu}_{1}} \\
\bar{d}_{2} & :=\frac{\tilde{\beta} \tau e_{2}\left(2 \nu_{2}-2 d_{2} \nu_{2}+\left(2 \nu_{2}-2 d_{2} \nu_{2}-\nu_{1}+d_{1} \nu_{1}\right) \tilde{\beta}\right.}{e_{1} \bar{\tau} \bar{\nu}_{2}}
\end{aligned}
$$

and define

$$
\begin{aligned}
& h_{0}:=\left[\begin{array}{l}
0 \\
1
\end{array}\right], h_{1}:=\left[\begin{array}{c}
\nu_{1} d_{1} \\
\nu 1
\end{array}\right], h_{2}:=\left[\begin{array}{c}
\nu_{2} d_{2} \\
\nu 2
\end{array}\right], h_{3}:=\left[\begin{array}{l}
1 \\
1
\end{array}\right] \\
& \bar{h}_{0}:=\left[\begin{array}{l}
0 \\
1
\end{array}\right], \bar{h}_{1}:=\left[\begin{array}{c}
\bar{\nu}_{1} \bar{d}_{1} \\
\bar{\nu} 1
\end{array}\right], \bar{h}_{2}:=\left[\begin{array}{c}
\bar{\nu}_{2} \bar{d}_{2} \\
\bar{\nu} 2
\end{array}\right], \bar{h}_{3}:=\left[\begin{array}{l}
1 \\
1
\end{array}\right]
\end{aligned}
$$

\subsection{Quadratic $C^{1}$ reparameterization $\eta$}

We consider three consecutive quadratic spline segments $f^{i-1}, f^{i}$ and $f^{i+1}$ with rational weights $1, w_{j}, 1, j \in\{i-1, i, i+1\}$ that are $G^{1}$-connected with geometric continuity scalars $\beta_{i}$, respectively $\beta_{i+1}$. The formulas allow for setting new continuity scalars $\tilde{\beta}_{i}, \tilde{\beta}_{i+1}$, for example by averaging old ones. For segment $i$, we define two rational quadratic reparameterizations, $h^{i, j}, j \in\{0,1\}$ with homogeneous coefficients

$$
\begin{aligned}
& {\left[\begin{array}{l}
0 \\
1
\end{array}\right], a_{j}^{i}\left[\begin{array}{c}
\frac{1}{2} \\
1
\end{array}\right],\left[\begin{array}{l}
1 \\
1
\end{array}\right],} \\
& a_{0}^{i}:=\frac{2\left(1+\frac{1}{\tilde{\beta}_{i}}\right)}{w_{i} \frac{1}{\beta_{i}}+w_{i-1}+\frac{1}{\beta_{i}}+1}, \quad a_{1}^{i}:=\frac{2\left(1+\tilde{\beta}_{i+1}\right)}{w_{i} \beta_{i+1}+w_{i+1}+\beta_{i+1}+1} .
\end{aligned}
$$

Then the left piece of the reparameterization is $\eta^{i, 0}:=h^{i, 0}(u / 2)$ and the right is $\eta^{i, 1}:=h^{i, 1}(1 / 2+u / 2)$. Let $\eta_{k}^{i, j}$ be the corresponding homogeneous control points. To guarantee $C^{1}$ continuity between $\eta^{i, 0}$ and $\eta^{i, 1}$, we overwrite the common point by $\eta_{2}^{i, 0}=\eta_{0}^{i, 1}:=\frac{\eta_{1}^{i, 0}+\eta_{1}^{i, 1}}{2}$. Lemma 2 states that also the connection between original segments is also smooth. 


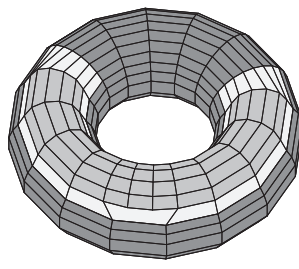

(a) mesh

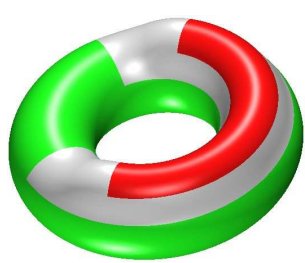

(b) $C^{1}$ ensemble

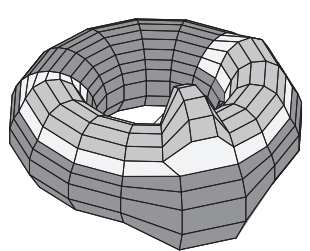

(c) edited mesh

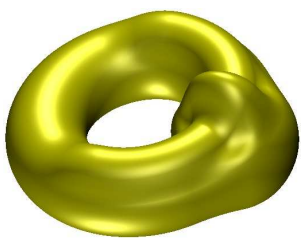

(d) $C^{1}$ surface

Figure 14: $C^{1}$ torus ensemble with differing $\beta_{i}$ and T-joints.

Lemma 2 (homogeneous $C^{1}$ reparameterization) Let $f^{i} \circ \eta^{i, 0}, f^{i} \circ \eta^{i, 1}$ be the two-piece reparameterization of the segment $f^{i}$. Then the quartics $f^{i-1}\left(\eta^{i-1,1}\right)$ and $f^{i}\left(\eta^{i, 0}\right)$ are $C^{1}$-connected in homogeneous space with scalar $\tilde{\beta}_{i}$. 


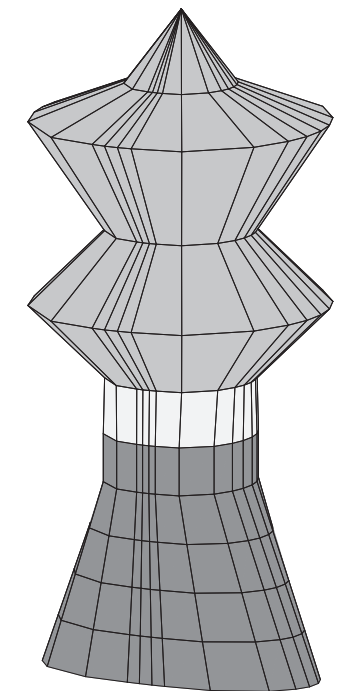

(a) combined mesh

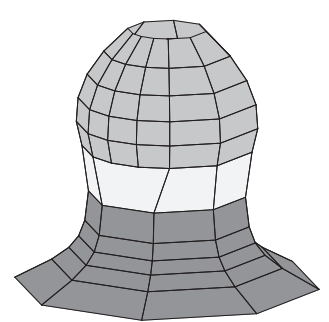

(e) combined mesh

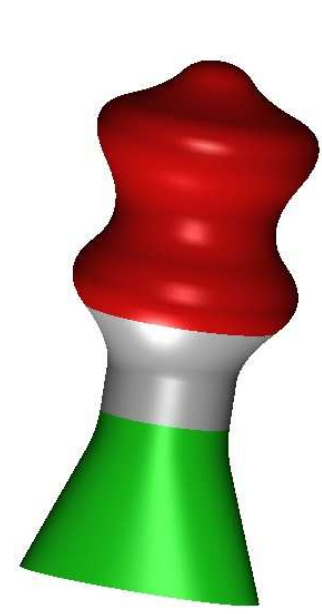

(b) combined surface

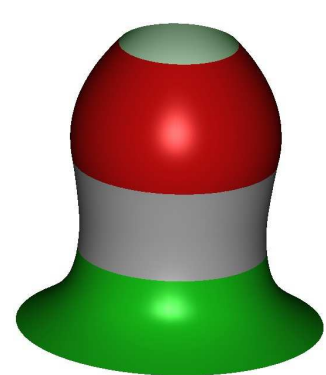

(f) combined surface

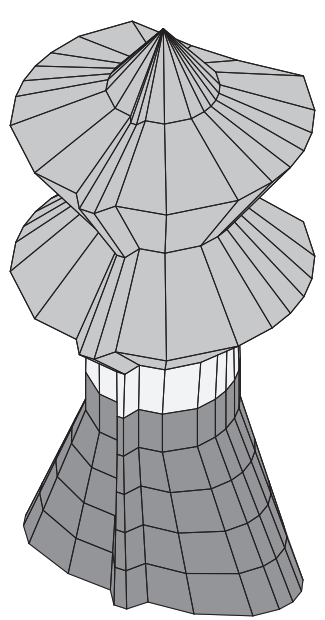

(c) edited mesh

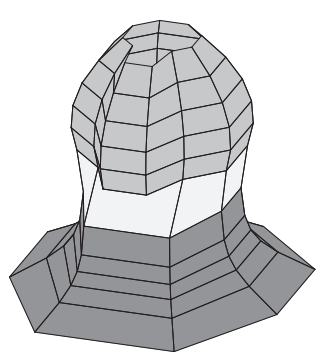

(g) edited mesh

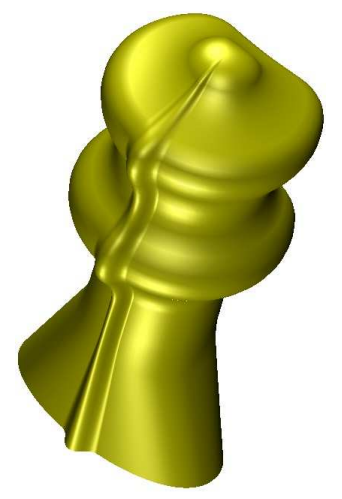

(d) $C^{1}$ ensemble

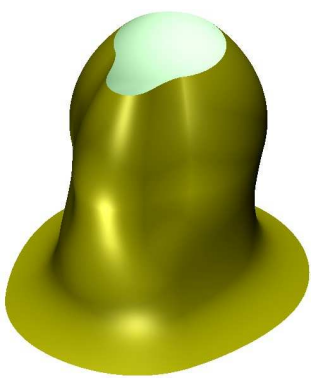

(h) $C^{1}$ ensemble

Figure 15: Unified $C^{1}$ representation of degree $(4,2)$. (a) Surface of revolution with $C^{1}$ quadratic spline profile curve (top) and bi-2 $C^{1}$ spline (bottom). (e) Surface of revolution with parabola as profile curve (top) and with hyperbola (bottom). Note the T-joint. 